\title{
Mental search processes in problem solving
}

\author{
JAMES SHANTEAU \\ Kansas State University, Manhattan, Kansas 66506 \\ and \\ GARY H. McCLELLAND \\ University of Colorado, Boulder, Colorado 80302
}

\begin{abstract}
A new approach to problem solving was applied to multisolution problems in a memory search task. Subjects memorized a list of eight four-letter foods, and then searched mentally through the list for answers to questions. The times between successive answers (IRTs) were recorded along with the answers themselves. This allowed a comparison of two possible memory search strategies: (1) sampling with replacement, and (2) sampling without replacement. The results were largely in agreement with the sampling-without-replacement strategy. However, a more detailed breakdown of the data revealed that most subjects searched through the list in a rigid serial order. Further, an analysis of questions with identical answers showed that the IRTs were very nearly additive. This led to an additive time component model based on the independent summation of (a) read-in time, (b) memory-search time, (c) decision-making time, and (d) response-output time. This approach appeared generally more satisfactory than previous attempts to account for problem-solving behavior.
\end{abstract}

Prompted by the work on memory scanning by Sternberg $(1966,1969)$, the role of memory search processes has recently received considerable attention. While most research has dealt with variants of Sternberg's memory task. there has been less work on higher mental processes. The goal of this paper is to analyze the role of mental search processes in thought. Specifically, the purpose is to investigate the reaction time components involved in the search for and the discovery of solutions to problems.

The importance of search processes in thinking and problem solving has been historically recognized (e.g. . Johnson. 1944). Miller. Galanter, and Pribram (1960) note that the "general conception-that thinking. problem solving, concept attainment can be modeled after the search process-is a familiar one to psichologists" (p. 163). They go on to add "that problem-solving can be represented as searching through a large set of possibilities until we find one that solves the problem" (p. 167). Similarly, Campbell (1960) argued that creative thought involves

This work was done at the University of Michigan during the senior author's tenure in U.S. Public Health Service Postdoctoral Fellowship 1 F02 MH-36963-01 from the National Institute of Mental Health, and during the second author's tenure in U.S. Public Health Service Training Grant GM 0123108 from the National Institute of Mental Health. The preparation of this paper was supported by U.S. Public Health Service Grant MH 26002 from the National Institute of Mental Health. The authors wish to thank James Greeno and Brown Grier for their advice during the initial stages of work. The authors also wish to thank Keith Smith for suggesting the confidence interval test for tau. Requests for reprints should be sent to James Shanteau. Department of Psychology. Kansas State University. Manhattan. Kansas 66506. a blind search for answers. Such ideas were applied by Restle (1969; Restle \& Davis. 1962) in a formal mathematical approach to problem solving. According to Restle (1969). problem solving is a search process made up of several steps. Each of these steps leads to a search which terminates with a discovery.

Restle translated this view of problem solving into a mathematical model by making two assumptions about the search process. These assumptions are. first. that problems are solved by a series of independent searches or steps and, second, that each step proceeds in an all-or-none fashion. The solution latency is then the sum of the latencies of the various steps. This leads to a geometric distribution of search times for a single step. and a generalized gamma distribution for several steps (McGill, 1963). This model was. found by Restle and Davis (1962) to provide a close fit to the latency distributions for three "logic twister" problems. It also provided reasonable values for the number of steps required to solve each problem.

Anderson (1969) challenged the adequacy of this analysis. and instead offered an alternative experimental test of Restle's model. He proposed a task in which subjects make an explicit mental search through a memorized list looking for words with certain "key" features. This task incorporates leatures of both Sternberg's (1966) memory scanning task and Bousfield and Sedgewick's (1944) word production task. For instance, subjects in this study memorized the list shown in Table 1. They were then asked questions about the list such as "Which come 
Table 1

List of Eight Memorized Words and Set of Sample Questions and Answers

List of Words: RICE, CAKE, TUNA, BEEF, FISH, PEAR, LIME, VEAL

\begin{tabular}{cl} 
No. & Question \\
\hline 0 & Which come from pigs? \\
1 & Which have second letter "A"? \\
2 & Which have letters "E" and "I"? \\
3 & Which come from plants? \\
4 & Which have bones? \\
5 & Which have first letter which rhymes with "ME"? \\
6 & Which are not fruits? \\
7 & Which do not rhyme with "DISH"? \\
8 & Which have letters in common with "ITEM"?
\end{tabular}

Answer

(None)

CAKE

RICE, LIME

RICE, PEAR, LIME

TUNA, BEEF, FISH, VEAL

CAKE, TUNA, BEEF, PEAR, VEAL

RICE, CAKE, TUNA, BEEF, FISH, VEAL

RICE, CAKE, TUNA, BEEF, PEAR, LIME, VEAL

RICE, CAKE, TUNA, BEEF, FISH, PEAR, LIME, VEAL

from plants?" (see Table 1 for other examples). The three answers (RICE, PEAR, and LIME) point out a unique feature of this task. That is, the questions can have several solutions. In contrast, the problems used in most previous research have had single solutions or answers (Davis, 1966).

One aim of this paper is to examine the assumptions of Restle's model. For the Anderson task, this model suggests that solutions are found by randomly sampling from the list of words. After each word is sampled, the all-or-none assumption implies that the word is returned to the pool of possible answers. That is, inappropriate words are rejected and returned to the pool, while appropriate words are used as answers and then returned to become inappropriate answers for later samples. This, in short, is equivalent to the familiar strategy of sampling with replacement. The expected pattern of results for this strategy have been derived in the Appendix, and are shown in the left panel of Figure 1. For a total number of solutions, $k$, the sampling-with-replacement strategy implies that each

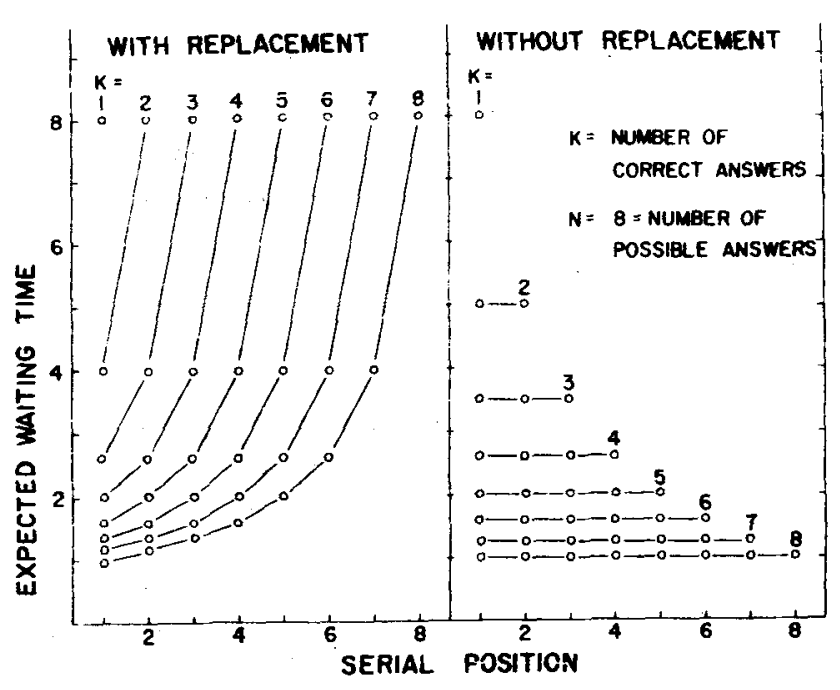

Figure 1. Theoretical expected waiting times between responses for search strategies of sampling with and without replacement. (Vertical axis units are arbitrary.) successive solution requires more time than the previous one. That is, there should be a steady increase in interresponse times (IRTs) across serial position. Evidence for such a search strategy has been reported in both problem solving studies (Restle \& Davis, 1962) and memory search studies (Bousfield \& Sedgewick, 1944; Indow \& Togano, 1970; Metlay, Handley, \& Kaplan, 1971).

As an alternative to sampling with replacement, Anderson (1969) suggested that the search process might proceed by a strategy of sampling without replacement. That is, once a word is sampled and found to be either an appropriate or an inappropriate answer, it is eliminated from the pool of possible answers. The predictions for this strategy are also derived in the Appendix, and are shown in the right panel of Figure 1. For a question with $k$ solutions, the times between the $\mathrm{k}$ successive solutions should all be the same. While evidence for such a search strategy is scarce, Indow and Togano (1970) do report some suggestive data.

The difference in the two panels of Figure 1 demonstrate one advantage of the multiple-solution task. If only a single solution had been reported, the results would include just the points for the first serial position. Obviously, discrimination of the two search strategies would then be very difficult. In contrast, the two strategies are quite easy to discriminate with multiple solutions. This reflects the fact that the with-replacement strategy samples from a pool of answers of constant size, while the withoutreplacement strategy samples from a pool which dininishes with every sample.

\section{METHOD}

Subjects thoroughly memorized a list of eight four-letter foods. and then answered questions about the list. Since most questions had multiple correct answers, the interresponse times between answers can be used to test the random sampling models.

\section{Apparatus}

A Digital Equipment Corporation LINC.8 laboratory computer was used to present stimuli and to time and record responses. The instructions and stimuli were displayed on a CRT screen. The subject responded by typing on a Teletype keyboard located directly in front of the screen. 


\section{Procedure}

There were three basic sections, the first two of which served as preparation for collecting the critical data in the third section. The first section consisted of instructions and practice on the operation of the Teletype, as well as training on the list of eight four-letter foods. Initially, the list in Table 1 was presented in two groups of tour words. The subject was then given a subset of four words in random order and asked to type in the missing four. The missing four words were displayed in random order following the subject's response. This was repeated 12 times, and was done to train subjects on the list without instilling any specific order.

The second section contained recognition questions of the sort used by Sternberg (1966). The subject responded to questions of the form "IS IN THE LIST?" where the blank was filled either by a word from the eight-word list or by a four-letter food word not on the list. The subject was instructed to respond as rapidly as possible by pressing one of two keys on the Teletype marked "YES" and "NO." The purpose of the recognition task was to ensure that the subject had adequately memorized the list and to give additional practice with the Teletype.

The third section contained the critical search questions and is the only section reported here. Each trial began with the display of a question, such as, "Which have either letters A or B?" The subject then typed as rapidly as possible the first letter of each word that correctly answered the question. In this case, the subject would type $C, T, B, P, V$, which indicates that CAKE. TUNA, BEEF, PEAR, and VEAL are appropriate answers. When the subject was through giving answers, he pushed the "RETURN" key. This signaled that his search for answers was complete and initiated the next trial after a delay of $7 \mathrm{sec}$.

The order of the responses along with their latencies were recorded. The tirst latency was measured from the time the question was displayed until the first response. All other latencies were measured from the previous response.

An effort was made to increase the reliability by having repeated measures. However, because the subject might remember previous answers, it was not feasible to replicate the same question. Greater reliability was achieved instead by asking different questions with the same answers. For example, six questions were asked which all had the same five answers, CAKE, TUNA, BEEF, PEAR, and VEAL; four of these questions are shown in the right part of Figure 4. Five analogous sets of questions were constructed with three to eight identical answers; questions with less than three answers are not useful in distinguishing between the models. To keep subjects from recognizing the similarity of the answers across questions, the questions were randomly ordered and filler questions werc also included.

\section{Scoring}

The four most representative questions for each answer subset were selected for the tinal analysis. When a subject failed to agree with an answer given by the other subjects, the value of the mean was substituted for his latency. Six percent of the data was replaced in this fashion. In addition, a latency for a subject which exceeded the next closest latency for other subjects by more than $4 \mathrm{sec}$ was considered as an outlier and replaced by the mean. This replaced $0.5 \%$ of the data.

\section{Subjects}

The subjects were 15 undergraduate females from the University of Michigan who knew how to type. They were run for approximately $90 \mathrm{~min}$ and were paid $\$ 3$.

\section{RESULTS}

The mean interresponse times (IRTs) are plotted in Figure 2 . These values were obtained by averaging over the sets of four questions with common answers. The most notable feature of the curves is their

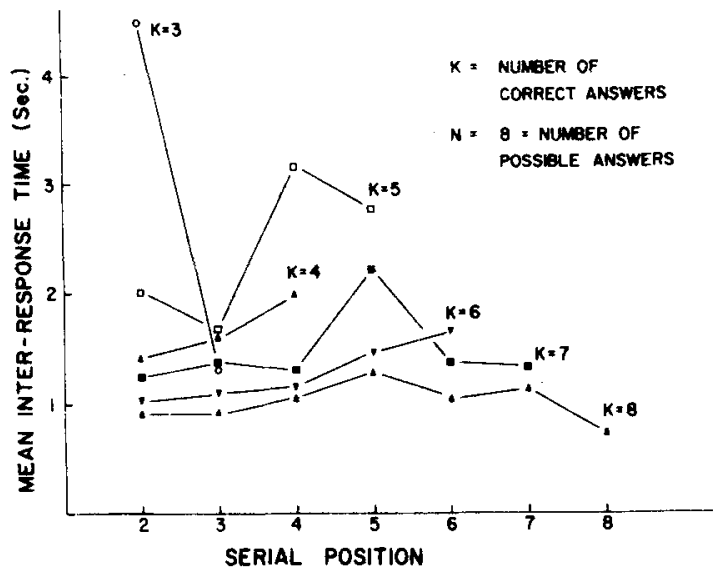

Figure 2. Obtained IRTs averaged across questions with similar answers for Sets 3 through 8 . (Initial IRTs have been omitted for reasons given in text.)

flatness. This was predicted by the sampling-withoutreplacement strategy, and there is certainly little to suggest the upward sweep predicted by the with-replacement strategy. Overall, the data appear to be generally in accord with the withoutreplacement strategy.

However, three of the curves in Figure 2 show marked deviations from horizontal. Accordingly, the curves for the three, five, and seven answer sets were broken down into the four questions making up each set. The results of this breakdown are shown in Figures 3, 4, and 5. As can be seen, the deviations from horizontal were consistent across different questions.

\section{Analysis of Response Order}

The consistency of the deviations led to a reexamination of the data. As a first step, the output order of the answers was examined. If the subjects were indeed randomly sampling from the list of foods, then there should be no discernible order to their

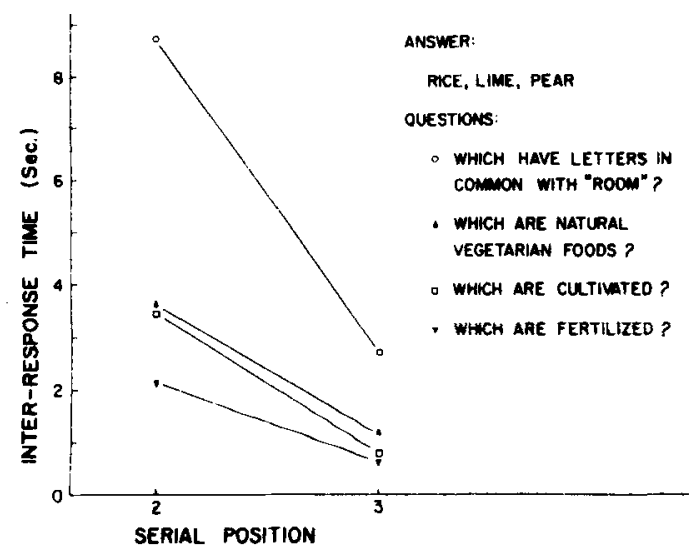

Figure 3. Mean IRTs for questions with same three answers. 


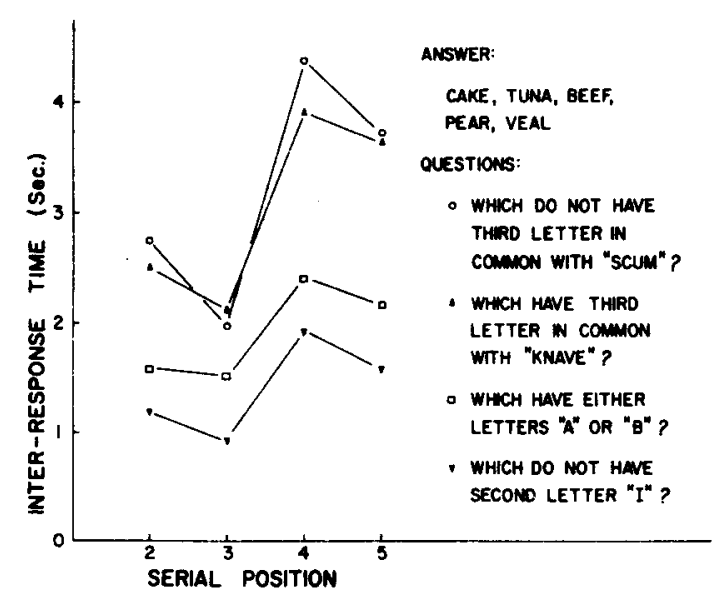

Figure 4. Mean IRTs for questions with same five answers.

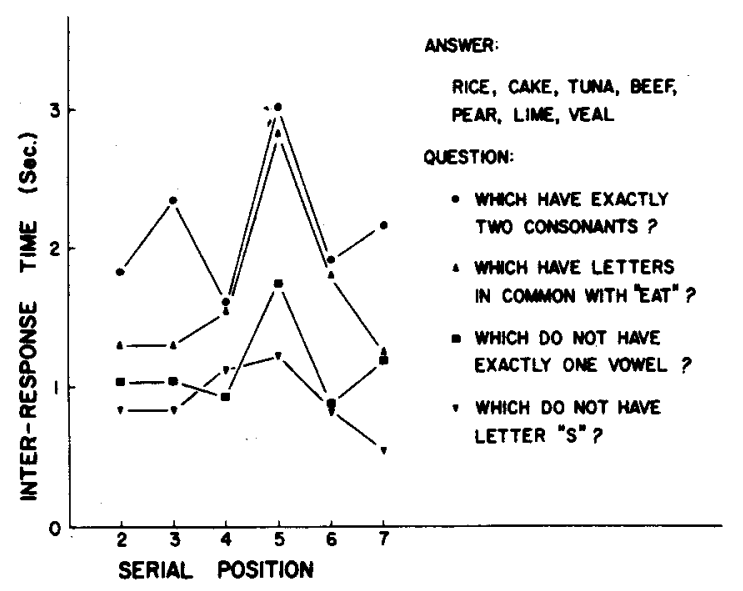

Figure 5. Mean IRTs for questions with same seven answers. (Filled points represent logically similar questions.)

answers. However, an examination of each subject's answers revealed systematic orderings. To determine the extent of this ordering, the output order for each question was compared by a rank-order correlation to the order which each subject gave most consistently to the eight-answer questions. A weighted average tau was computed for each subject, with the weight based on the number of answers given to each question. Using a conservative test based on an overestimate of the variance, all taus were significantly different from zero $(p<.05)$. In fact, the values for most subjects were quite close to a perfect $1.0 ; 9$ of 15 subjects had taus above .9 , while only 2 subjects had values below .8. Further, the values for 6 subjects would have been even higher except that they apparently modified their orders part way through. Thus, the taus for these subjects are underestimates of their true consistency.

The order in which subjects first saw the words is shown in Table 1, and most subjects seem to have been strongly influenced by this order. In fact, each word was in its original serial position for an average of 9.0 subjects. This ranged from 13 subjects who started their list with RICE down to 5 subjects who ended their list with VEAL. This consistency occurred in spite of the fact that the subjects saw the original order only once, and even then saw only half the words at a time. Therefore, subjects apparently have a strong tendency to use some serial order, and it seems doubtful that any procedural modification would have changed that.

\section{Analysis of Parallelism}

As exemplified by Figures 3-5, the curves appeared to be roughly parallel to each other. To examine this further, analyses of variance were performed on all six answer sets, with serial position and questions as the factors. The parallelism observation was substantiated by nonsignificant interactions in all but Set 3 in Figure $3[\mathrm{~F}(3,42)=29.67 / 3.75=7.91$; $\mathrm{p}<.05]$ and Set 7 in Figure $5[\mathrm{~F}(15,210)=1.28 / .71$ $=1.80 \mathrm{]}$.

In addition to the lack of interactions, the analyses revealed that both main effects were significant for most answer sets. The serial position effect was significant for all except Set 4 , and the question effect was significant for all except Set 8 . Thus, there were clear differences in the IRTs as a function of both the different questions asked and the serial position of the answers.

\section{Proposed Model}

The previous analyses indicate that neither search strategy is appropriate for the present results. This led to the proposal of a new model of problem solving based on four stages or processes. The sequence of processes is shown in flow-chart form in Figure 6. Each process is assumed to contribute an independent time component, which then adds up to the total IRT. The first component is the time to READ IN question $k\left(I_{k}\right)$; this occurs only prior to the first answer. Next

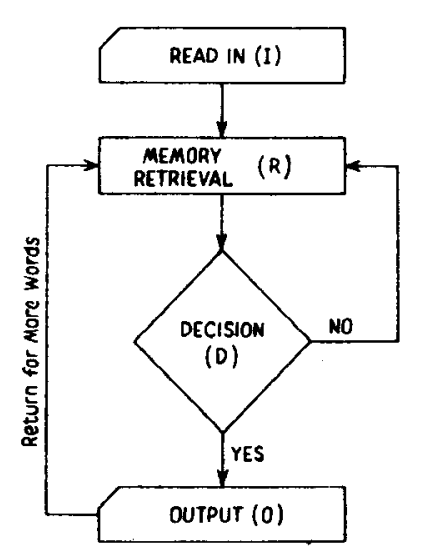

Figure 6. Flow chart of proposed additive time component model. (Arrows give assumed sequence of problem solving processes.) 
is the sum of the times taken to RETRIEVE word $i$ from the list in memory $\left(R_{i}\right)$ and to make a DECISION about whether that word is an answer to the question or not $\left(D_{k}\right)$; this sum continues until an appropriate answer, $n$, is found. Finally comes the OUTPUT of the response $\left(\mathrm{O}_{\mathrm{n}}\right)$. This is followed by retrieval of the next word on the list, and the sequence of processes is repeated until all answers are found.

The rationale behind this model can be clarified by considering the pattern of IRTs in the figures. In Figure 5, for instance, the curves all peak at the fifth IRT. Given the initial word order, this is where FISH would be expected. However, FISH is the only word which is not an appropriate answer for this set of questions. Thus, the peak apparently represents the time taken to retrieve and decide that FISH is not an answer, and then to retrieve and decide that the next word. PEAR, is an answer. This shows up in the flow chart as an extra loop because of FISH. This same correspondence between IRT peaks and omitted words was also found in other sets of questions.

It is possible to evaluate the processing-time components based on the pattern of IRTs. For instance, there appeared to be slight differences in RETRIEVAL times for various words. These showed up as small, but consistent, ups and downs in the IRT curves where there were no intervening words. An examination of the eight-answer set revealed that the middle IRTs were slightly longer. This apparently means that some words are easier and quicker to retrieve from memory than others. Also, there were marked differences in DECISION times for various questions. These showed up as consistent differences in the relative elevation of the curves. The higher curves are suggestive of more difficult questions, which require longer decision times. Thus, the height of each curve apparently reflects the relative difficulty of the decision processes required to answer the question.

To further illustrate the importance of DECISION processes, consider the first and third curves in Figure 5 (represented by filled squares and circles, respectively). These two questions are logically equivalent for the list of words. However, the question, "Which do not have exactly one vowel?" (filled squares), is stated negatively, and can be answered in one of two ways. The subject can search for words with zero, two, three, or four vowels and answer every time a word is found. More likely, the subject searches for words with one vowel and gives an answer when anything else is found. In either case, the logical analysis is more complex than that needed to answer "Which have exactly two consonants?" (filled circles). Here, the subject need only search for words with two consonants (or two vowels) and give these words as answers. Similarly, a linguistic analysis would also suggest that negative questions are more complex than positive questions. The IRTs in
Figure 5 , however, reveal quite a different picture. The positive question takes anywhere from .7 to $1.3 \mathrm{sec}$ longer per answer than the negative question. Thus, the decision time for the positive question is roughly $1 \mathrm{sec}$ longer than the decision time for the negative question. While these conclusions are only tentative, they suggest that logical and linguistic analyses may not be indicative of the psychological difficulty of deciding on answers.

It was not possible to conclude much about READ IN and OUTPUT times with the present design. Indeed, the initial response was specifically excluded in the analyses to avoid the contaminating effect of reading times. However, post hoc analyses of the initial latencies revealed times varying between 3 and $11 \mathrm{sec}$. Roughly half this time can be attributed to reading time, with the remainder due to the other processes required to get the first answer. In comparison, a post hoc analysis of output times revealed little of any interest. Such times reflect mainly motor components, and appeared to have little influence on the shape of the IRT curves.

An important advantage of the proposed model is that the perceptual/motor processes of READ IN and OUTPUT can be separated from the cognitive processes of RETRIEVAL and DECISION. In previous research, the perceptual/motor and cognitive processes have frequency been confounded.

\section{Mathematical Model}

The proposed model can be stated in more precise mathematical terms. The first latency $\left({ }_{1} I R T_{k n}\right)$ for word $\mathrm{n}$ as an answer to question $\mathrm{k}$ is:

$$
{ }_{1} \mathrm{IRT}_{\mathrm{kn}}=\mathrm{I}_{\mathrm{k}}+\sum_{\mathrm{i}=1}^{\mathrm{n}}\left(\mathrm{R}_{\mathrm{i}}+\mathrm{D}_{\mathrm{k}}\right)+\mathrm{O}_{\mathrm{n}}
$$

The summation covers the time to retrieve and decide whether the first word is an answer or not, and if it is not, to retrieve and decide for more words until an answer is found. The first and last terms correspond to read-in and output times for the question and answer, respectively. For answers after the first, Equation 1 can be simplified by eliminating the read-in time, $I_{k}$. For instance, the time ${ }_{2} I R T=T_{k m}$ to find the second answer $m(m>n)$ to question $k$ is:

$$
{ }_{2} \mathrm{IRT}_{\mathrm{km}}=\sum_{\mathrm{i}=\mathrm{n}}^{\mathrm{m}}\left(\mathrm{R}_{\mathrm{i}}+\mathrm{D}_{\mathrm{k}}\right)+\mathrm{O}_{\mathrm{m}}
$$

This states that the second (and any subsequent) IRT equals the sum of the retrieval and decision times needed to find the next answer, plus the time taken to output the response.

One technical comment on the model should be made at this point. There are two versions of the 
model depending on what is assumed about "no" decision times. The first possibility is that "no" decisions take the same time for all questions. This version of the model predicts strict parallelism in the IRT curves for any set of questions with common answers. Such parallelism was in fact approximated for all but two of the six sets of questions. An alternative view is that the time for "no" decisions depends on the difficulty of the question. This version of the model predicts that higher curves should have more exaggerated peaks than lower curves. For every "no" decision time for an easy question, there should be an even greater "no" time for a more difficult question. Since answers are given only after "yes" decisions, these differences in "no" times accumulate until the next answer is given. The interactions in Figures 3 and 5 are. in fact, of the form predicted by the second version of the model. While both versions have thus received some support in the present study, more precise comparisons must await later research.

\section{DISCUSSION}

The purpose of this study was to investigate the mental search processes used to solve multiplesolution problems. Two random search strategies were originally considered: Restle's sampling-withreplacement strategy and Anderson's samplingwithout-replacement strategy. Both strategies assume that subjects are randomly searching through the list. but disagree on the method of the search. While the sampling-without-replacement strategy was closer to the data, the results indicate that neither strategy was appropriate. Instead of randomly searching through the list, subjects searched in a systematic serial fashion. This suggests two issues which need further claboration. The first deals with the appropriateness and generalizability of the multiple-solution task used here. The second concerns the proposed model and its relation to other models of reaction time.

\section{Multiple Solution Task}

The generalizability of the proposed task seems very limited at tirst glance. After all, there are not many instances where we first memorize a list of words, and then answer questions about just that list. However, the answers to many questions do in fact come from some subset or "list" of possible answers. For example. the answer to "Which days of the week are spelled with a T?" obviously involves a search through the "list" of the seven days of the week. This list is readily available to us, and yet it was not prompted in advance. Similarly, the answer to "Which of your past houses had front doors which open to the left?" (adapted from Norman, Note 1) also involves a clearly defined list. Thus, a search through a list seems to be a natural way of solving many types of problems.

Along this line, the findings of Indow and Togano (1970) may be of some illustrative value. They asked subjects to name Japanese cities from north to south and found results compatible with the present findings. However, when they simply asked subjects to name $J$ apanese cities. they found results more like the sampling-with-replacement strategy. While this finding was not followed up (Indow, personal communication, 1973), it does suggest that the question itself may prompt an ordered search for answers. It may well be that some questions lead to one type of solution strategy, while other questions are better described by another strategy.

In addition to the present application, the multiple-solution task should be valuable in evaluating problem solving processes in a wider variety of situations. In this regard, it may prove useful to follow the classification of questions suggested by Anderson (1969). According to his breakdown, questions such as "Which have second letter I and fourth letter E?" are conjunctive, since more than one feature is examined. In comparison, questions such as "Which end in letter E?" are disjunctive, since only one feature is relevant. The answer to the latter question presumably involves a direct comparative process, whereas the former question requires an additional short-term memory storage to hold the results of the two comparisons. This additional memory process can be directly evaluated since the two questions above have the same answers. RICE and LIME. Therefore, any differences between the IRTs could only come from the different processes required to obtain the answers.

Similar procedures may prove profitable in analyzing the processes used to answer semantic questions, such as "Which are smaller than a peach?" To answer this question, the size of peach must be compared to the sizes of the eight foods. This size information presumably comes from long-term semantic memory. Since this question also has the same answers as the previous questions, it should prove possible to estimate the time taken by semantic memory processes. Finally, such questions as "Which words have second letters in common and also have fourth letters in common?" can be considered. Such questions depend on finding interrelated answers. Instead of looking for specitic features, the answer comes from looking for a match in a pairwise comparison of letters across words. Such a construction of answers is especially relevant to thought processes (Restle, 1969), and the present procedures may prove useful in evaluating these processes. For instance, this last question also has the same answers, RICE and LIME, and this should allow direct analysis of the processing times involved.

\section{Previous Models}

The second general comment concerns the relation of the proposed model to earlier formulations. Most previous multiple-response models have the property of an extinction chain (McGill, 1963). That is, 
responses become less frequent as more responses are given. Probably the best known example of this type of model is the negative exponential. This was first proposed by Bousfield and Sedgewick (1944) for word production latencies. Recent discussions of such models have appeared in Indow and Togano (1970), Metlay et al. (1971), and Restle and Davis (1962). Of more immediate interest, the sampling-withreplacement strategy in Figure 1 is an instance of this type of model. Of course, the present results are incompatible with any such model.

The study by Metlay et al. (1971) is particularly relevant here. They had subjects memorize lists such as all U.S. Presidents. They then asked subjects to give the names of Presidents with a specific letter, such as E. In each of three cases, the cumulative latency distribution was fit by a negative exponential curve. Since the task is quite similar to the one proposed here, this finding would appear to be contradictory to the present results. However, there are several differences which make comparisons between studies difficult. Probably the most important is that their subjects were unable to remember all the names. In contrast, subjects here overlearned the list of foods. This suggests that the familiarity of the list may determine the type of solution strategy used.

Most recent work on reaction time has centered around additive time component models. That is, times for various psychological processes are assumed to make separate contributions to the total latency. For example. Stone (1960) proposed a model for choice reaction times based on three additive components: a neutral (input) time, a decision time, and a movement (response) time. A similar three-component model was advanced by Falmagne and Theios (1969) with a template formation stage, a comparison stage, and a response stage. A recent application of this model was contained in the study by Theios, Smith, Haviland, Traupmann, and Moy (1973) on the Sternberg (1966) memory scanning task. In this application, the comparison time depended on differences in memory load and organization, and so the second stage might best be viewed as a combination memory retrieval and decision component.

While sharing several obvious similarities with these previous models, the proposed model has two important distinctions. First, the present application of an additive composition model to problem solving (or question answering) appears to be novel. Most previous applications have been either to choice reaction time tasks or to simple memory scanning tasks. Thus, the present application marks an extension of the use of additive composition models. A second distinction involves the use of separate RETRIEVAL and DECISION components in the present model. In previous formulations, these components have either been combined or one of the components has been ignored. While this may have been adequate for simple reaction time tasks, separate retrieval and decision components are required to account for the present results. Of course, it may prove useful in future work to subdivide the proposed components even further to represent, for example, short-term and long-term memory retrieval processes.

Finally, some comment should be made on the additive factor method introduced by Sternberg (1969). The main feature of this method "is the search for non-interacting effects of experimental factors on mean RT" (p. 276). This leads to analyses of parallelism which are quite similar to the present techniques. Indeed, Sternberg (1969, Figure 6) discussed a four-factor model which bears a striking resemblance to the model proposed here. While related to the additive factor model, the approach used here is different in several ways. For one thing, the present approach involves a multiple-response task, whereas the Sternberg approach is concerned with single-response tasks. Another difference is that Sternberg makes use of parallelism to compare latencies under various experimental conditions, e.g., normal and degraded stimuli. In contrast, the analysis of parallelism here involves chains of response latencies. It might prove useful to combine the two approaches, e.g., by using degraded stimuli in a multiple-response task. This would allow an examination of the effect of experimental factors, such as stimulus degradation, on the varions processes of problem solving.

\section{APPENDIX \\ Two Memory Models for a Multiple-Solution Problem-Solving Task}

Let $A=\left\{a_{1}, a_{2}, \ldots, a_{n}\right\}$ be a specified set of responses from which the answers are to be selected. Also, let $A_{i}^{*}=\left\{a_{i 1}^{*}, a_{i 2}^{*}, \ldots\right.$, $\left.a_{i k}^{*}\right\}$ be a subset of $A$ such that the $k$ elements of $A_{i}^{*}$ are appropriate or correct answers for problem $\mathrm{i}$. Finally, $\mathrm{T}_{\mathrm{ij}}$ is a random variable (measured in units of the time necessary to sample one element from $A$ ) which represents the interresponse latency between the $j$-th and $j+1$ st element found from $A_{i}^{*}$. (For an excellent account of the discrete probability distributions used in the following derivations, see Johnson \& Kotz, 1969).

\section{Model I. Sampling With Replacement}

After presentation of problem $i$, set $A$ is searched until an element in $A_{j}^{*}$ is found. That element is given as a response and then replaced in set $A$ but removed from $A_{i}^{*}$. This search of $A$ continues until $A_{i}^{*}$ is empty.

After $j$ elements of $A_{i}^{*}$ have been found, the probability of finding the $\mathbf{j}+1$ st element on the next trial is $(\mathbf{k}-\mathbf{j}) / \mathbf{n}$ and remains so until the $j+1$ st is found. The waiting time ( $=$ the number of elements sampled which do not satisfy problem i) between the $j$-th and $j+1$ st elements of $A_{i}^{*}$ is described by the geometric distribution. so that

$$
P\left[T_{i}=t\right]=\left(\frac{k-j}{n}\right)\left(\frac{n-k+j}{n}\right)^{t}, \quad t=0,1,2, \ldots
$$


and

$$
E\left(T_{j}\right)=\frac{n-k+j}{n} / \frac{k-j}{n}=\frac{B}{k-j}-1 .
$$

so the expected total a aiting time. including the time to sample the $j+1$ st element in $A_{i}^{*}$. is $n(k-j)$. The predicted interresponse latencr curves for $\mathrm{n}=8$ and $\mathrm{k}=1.2 \ldots .8$ are presented in Figure 1.

\section{Mode II. Sampling Without Replacement}

After presentation of problem $i$. set $A$ is searched until an element in $A_{i}^{*}$ is found. That element is given as a response and then remosed from both $A$ and $A_{i}^{*}$. This search of $A$ continues until $A_{i}^{*}$ is empty.

The number of elements of $A_{i}^{*}$ found in t samples is given by the hypergeometric, so the waiting time until the selection of the $j$-th element satisfing the problem is given by the negative hypergeometric.

$$
\begin{aligned}
& P\left[\sum_{m=1}^{j} T_{m}=t\right]=\frac{\left(\begin{array}{c}
k \\
j-1
\end{array}\right)\left(\begin{array}{c}
n-k \\
t-j
\end{array}\right)}{\left(\begin{array}{c}
n \\
t-1
\end{array}\right)}\left(\frac{k-j+1}{n-t+1}\right) . \\
& t=j \cdot j+1 \ldots n-k+j \\
& E\left(\sum_{m=1}^{j} T_{m}\right)=\frac{j(n+1)}{(k+1)}
\end{aligned}
$$

So

$$
E\left(T_{j}\right)=E\left({ }^{j+1} \sum T_{m}\right)-E\left(\sum^{j} T_{m}\right)=\frac{n+1}{k+1}
$$

which does not depend on $\mathrm{j}$.

The theoretical values for $E\left(T_{j}\right)$ under Model $I I$ for $n=8$ and $k$ $=1.2 \ldots .8$ are given in Figure 1: a linear transformation has been applied to make the tro panels comparable.

\section{REFERENCE NOTE}

1. Norman. D. A. Remembrance of things past. Center for Human Information Processing Report No. 11. University of California. San Diego. 1970.

\section{REFERENCES}

Anderson. N. H. A search task. In J. F. Voss (Ed.), Approache's to thought. Columbus. Ohio: Merrill. 1969.

Bousfield, W. A.. \& Sedgewick. C. H. W. An analysis of sequences of restricted associative responses. Joumal of General Psichology. 1944. 30. 149-165.

CAMpbell. D. T. Blind variation and selective retention in creative thought as in other knowledge processes. Psichological Reviell. $1960.67,380-400$.

DAvis. G. A. Current status of research and theory in human problem solving. Psychological Bulletin, 1966. 66. 36-54.

Falmagne. J. C.. \& Theios. J. On attention and memory in reaction time experiments. Acta Psychologica. 1969. 30. 316-323.

InDow. T.. \& Togano. K. On retrieving sequence from long-term menory. Psychological Review. 1970. 77. 317-331.

Johnson. D. M. A modern account of problem solving. Psichological Bulletin. 1944. 41. 201-229.

Johnson. N. L.. \& Kotz. S. Distributions in statistics. Discrete distributions. New York: Houghton Mifflin. 1969.

McGill, W. J. Stochastic latency mechanisms. In R. D. Luce R. R. Bush. and E. Galanter (Eds.). Handbook of mathematical psichology (Vol. I). New York: Wiley. 1963.

Metlay. W.. Handley, A.. \& Kaplan. I. T. Memory search through categories of varying size. Journal of Experimental Psychology, 1971, 91. 215-219.

Miller. G. A.. Galanter. E. H.. \& Pribram. H. H. Plans and the structure of behavior. New York: Holt. 1960.

Restie. F. Mathematical models and thought. In J. F. Voss (Ed.). Approaches to thought. Columbus. Ohio: Merrill. 1969.

Restle. F.. \& Davis. J. H. Success and speed of problem solving by individuals and groups. Psychological Review. 1962. 69. $520-536$.

SternberG. S. High-speed scanning in human memory. Science. 1966. 153. $652-654$.

Sternaerg. S. The discovery of processing stages: Extensions of Donder's method. Acta Psychologica, 1969, 30. 276-315.

Stone. M. Models for choice reaction time. Psychometrika. 1960. 25. $251-260$.

Theios. I.. Smith. P. G.. Haviland. S. E.. Traupmann, J. \& MoY. M. E. Memory scanning as a serial self-terminating process. Jounal of Experimental Psicholog. 1973, 97. 323-336.

(Received for publication September 1974; revision received December 1974. ) 